\title{
Laguerre Runge-Kutta-Fehlberg Method for Simulating Laser Pulse Propagation in Biological Tissue
}

\author{
Chintha C. Handapangoda, Student Member, IEEE, Malin Premaratne, Senior Member, IEEE, \\ Leslie Yeo, and James Friend, Member, IEEE
}

\begin{abstract}
An efficient algorithm for solving the transient radiative transfer equation for laser pulse propagation in biological tissue is presented. A Laguerre expansion is used to represent the time dependency of the incident short pulse. The Runge-KuttaFehlberg method is used to solve the intensity. The discrete ordinates method is used to discretize with respect to azimuthal and zenith angles. This method offers the advantages of representing the intensity with a high accuracy using only a few Laguerre polynomials, and straightforward extension to inhomogeneous media. Also, this formulation can be easily extended for solving the 2-D and 3-D transient radiative transfer equations.
\end{abstract}

Index Terms-Biological tissue, discrete ordinates method, Laguerre expansion, Runge-Kutta-Fehlberg (RKF) method.

\section{INTRODUCTION}

I NVESTIGATIONS of short laser pulse propagation in biological media have attracted the attention of researchers over the last two decades [1], in particular, for light-based diagnostic and imaging purposes [1]. There has been growing interest in these optical techniques of late due to the fact that they are noninvasive and nonionizing [2]. The optical tomography for cancer detection [3] and noninvasive detection of diseases such as diabetes [4] are just two examples of the application of short laser pulses in medical treatment.

Photon transport through scattering and absorbing media, such as biological tissue, can be described by the radiative transfer equation (RTE) [5]-[7]. Biological tissue is a highly forward scattering medium with moderate amounts of photon attenuation. Thus, for the application of pulsed light interaction with biological tissue, the time-dependent radiative transfer equation can be used. A number of models for solving the steady-state RTE has been developed over the past four decades [8]-[14], ignoring the time dependency of the intensity profile. Some of these models include the discrete ordinates method [8], integral transformation techniques, and the $F_{N}$ method [11].

Manuscript received September 9, 2007; revised November 20, 2007.

C. C. Handapangoda and M. Premaratne are with the Advanced Computing and Simulation Laboratory (AXL), Department of Electrical and Computer Systems Engineering, Monash University, Clayton 3800, Vic., Australia (e-mail: chintha.handapangoda@eng.monash.edu.au; malin@eng.monash.edu.au).

L. Yeo and J. Friend are with the Micro/Nanophysics Research Laboratory (MNRL), Department of Mechanical Engineering, Monash University, Clayton 3800, Vic., Australia (e-mail: leslie.yeo@eng.monash.edu.au; james.friend@ eng.monash.edu.au).

Color versions of one or more of the figures in this paper are available online at http://ieeexplore.ieee.org.

Digital Object Identifier 10.1109/JSTQE.2007.913971
Siewert [12] and Larsen [14] have worked on the inverse-source problem where the source term is determined from the known angular distribution of radiation that exits the surface. Pomraning and Foglesong [13] have coupled the diffusion approximation and the radiative transfer equation for boundary treatment for the steady-state case.

In this paper, we deal with short pulses in tissue medium, and hence,we focus our attention on developing efficient and accurate techniques for time integration of the RTE. In the past, Dorn [15] has used the inverse problem of the time-dependent transport equation for modeling optical tomography. Larsen et al. [16] has carried out asymptotic analysis of radiative transfer problems, and Tarvainen et al. [17] have worked on finite element modeling of the coupled radiative transfer equation and diffusion approximation considering the time dependency.

However, only recently, researchers have started developing models for pulse propagation in biological tissue [19]. Existing methods for modeling short-pulse propagation through tissue include numerical methods [18], [20], [21] and semianalytical methods [22], [23]. Mitra and Kumar [6] compared several models for solving the transient RTE. Also, Tan and Hsu [19] developed an integral equation formulation to treat the general transient RTE, and Fleck used the Monte Carlo simulations [24]. Even though the Monte Carlo method is a flexible technique, due to its statistical nature, it requires tracing of a large number of energy bundles to obtain accurate and smooth solutions [18].

Kim et al. [21] have applied Chebyshev collocation techniques for solving the RTE [20]. Kim and Moscoso [2] has used Chebyshev expansion for the spatial discretization and CrankNicholson method for time marching. In many of the existing techniques, the short pulse is approximated by a square pulse [6], [18], which is not very accurate.

In this paper, we address some of the deficiencies in the aforementioned algorithms for solving the time-dependent radiative transfer equation. We do not limit our research to a particular pulse shape (e.g., square) but assume that it can be represented as a superposition of Laguerre orthogonal polynomials. The main advantage is that we could represent an arbitrary pulse shape using only a few Laguerre polynomials, considerably reducing subsequent computational overhead. The resulting equations are then solved using the Runge-Kutta-Felhberg (RKF) method. The extension of the model to inhomogeneous media is carried out without considerable increase in computational power.

This paper is organized in four sections. In Section II, we introduce the formulation of the proposed method. It provides an 


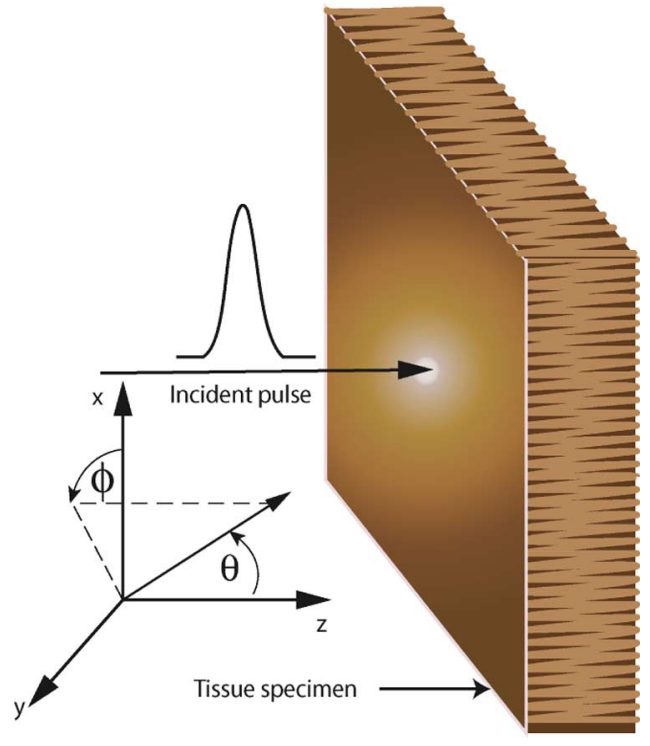

Fig. 1. Short light pulse incident on the biological tissue.

overview of the technique and contains three subsections with a detailed description and derivation of each step. Section III provides some numerical results obtained from this algorithm and a discussion on extending the proposed technique to more complex systems. We conclude in section IV with a discussion on relative advantages of the proposed method.

\section{Formulation}

Light propagation through biological tissue can be modeled by the radiative transfer equation given as [21]

$$
\begin{aligned}
& \frac{1}{v} \frac{\partial}{\partial t} I(z, u, \phi, t)+u \frac{\partial}{\partial z} I(z, u, \phi, t) \\
& -\frac{\sigma_{s}}{4 \pi} \int_{0}^{2 \pi} \int_{-1}^{1} P\left(u^{\prime}, \phi^{\prime} ; u, \phi\right) I\left(z, u^{\prime}, \phi^{\prime}, t\right) d u^{\prime} d \phi^{\prime} \\
& \quad+\sigma_{t} I(z, u, \phi, t)=F(z, u, \phi, t) \quad(1)
\end{aligned}
$$

where $I(z, u, \phi, t)$ is the light intensity, $(z, \theta, \phi)$ are the standard spherical coordinates as shown in Fig. $1, u=\cos \theta, t$ is the time variable, $\sigma_{t}$ and $\sigma_{s}$ are attenuation and scattering coefficients, respectively, and $\sigma_{t}=\sigma_{s}+\sigma_{a}$, where $\sigma_{a}$ is the absorption coefficient. The speed of light in the medium is denoted by $v$, $P\left(u^{\prime}, \phi^{\prime} ; u, \phi\right)$ is the phase function, and $F(z, u, \phi, t)$ refers to the source term. For applications where there is no source present inside the medium, $F(z, u, \phi, t)=0$. Without loss of generality, we consider this source-free condition in this paper to highlight important aspects of the algorithm.

Fig. 1 shows a short pulse incident on the tissue specimen from the left. In general, due to the index mismatch at the interface, radiation gets reflected. Even though the proposed method can handle such reflections at interfaces, due to the increased mathematical complexity in formulation that masks the main points of our algorithm, we limit our analysis to an indexmatched surrounding. This is because our focus is on presenting the main aspects of the proposed numerical solution tech-

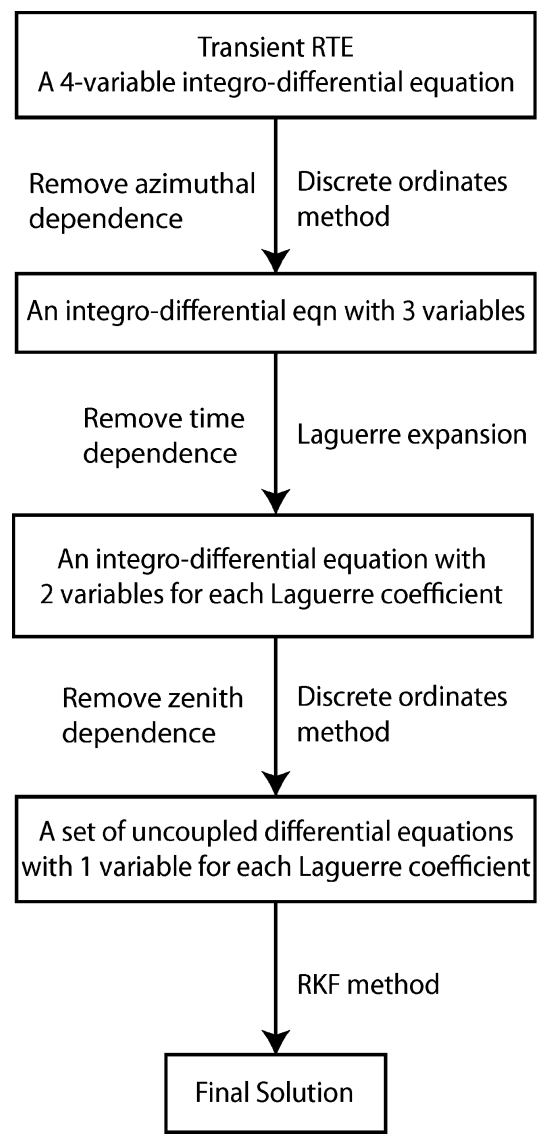

Fig. 2. Flowchart of the proposed method.

nique. However, a short discussion on index-mismatched case is provided in Section III-C.

The incident pulse is taken to be a point source incident at $z=0, u=u_{0}$, and $\phi=\phi_{0}$. Thus, the boundary condition can be written as

$$
I(z=0, u, \phi, t)=f(t) \delta\left(u-u_{0}\right) \delta\left(\phi-\phi_{0}\right)
$$

where $\delta(x)$ is the Dirac delta function [25] and $f(t)$ is the temporal profile of the pulse. Before proceeding to solve the RTE, the following substitution is used to map (1) to a moving reference frame with the pulse

$$
\begin{aligned}
\tau & =t-\frac{z}{v u} \\
\xi & =z .
\end{aligned}
$$

It is interesting to note that in the transformation (3), we calculate the delay in the pulse in a particular $u$ direction by using its projection in the $z$-direction (i.e., $z / u$ gives the actual propagation distance along the $u$-direction). Using (3) and (4) in (1),

$$
\begin{aligned}
& u \frac{\partial}{\partial \xi} I(\xi, u, \phi, \tau)+\sigma_{t} I(\xi, u, \phi, \tau) \\
& -\frac{\sigma_{s}}{4 \pi} \int_{0}^{2 \pi} \int_{-1}^{1} P\left(u^{\prime}, \phi^{\prime} ; u, \phi\right) I\left(\xi, u^{\prime}, \phi^{\prime}, \tau\right) d u^{\prime} d \phi^{\prime}=0 .
\end{aligned}
$$

We solve this transformed RTE numerically. Fig. 2 shows a flow chart of the proposed method. First, the azimuthal angle is 
discretized using the discrete ordinate method [8], [26], [27]. This results in a set of uncoupled three-variable integrodifferential equations, one for each quadrature point $\phi_{r}$. Then, a Laguerre expansion [28] is used to remove the time dependence in the main integro-differential equation. This results in a two-variable integro-differential equation for each Laguerre coefficient at each $\phi_{r}$. Next, discretization of the angle $\theta$ is performed using the discrete ordinates method [8], [26], [27]. This action results in a set of uncoupled single variable differential equations for each Laguerre coefficient. The reduced form of the RTE, subject to the boundary conditions given by (2) can, thus, be solved using the RKF method [29]. A detailed description of each stage is given later.

\section{A. Discretization of the Azimuthal Dependence}

First, the azimuthal angle $\phi$ can be discretized using the discrete ordinates method [8], [26], [27] by applying the Gaussian quadrature rule [30] for the integral that corresponds to $\phi$.

Gaussian quadrature is used to approximate an integral of the form $\int_{a}^{b} W(x) f(x) d x$, where $W(x)$ is the weight function and $f(x)$ is any arbitrary function, by a summation [30] as shown next

$$
\int_{a}^{b} W(x) f(x) d x \approx \sum_{j=0}^{N-1} w_{j} f\left(x_{j}\right) .
$$

We can choose weighting coefficients $w_{j}$, and abscissas $x_{j}$, so that the approximation in (6) is exact if $f(x)$ is a polynomial [30]. Even though one can generate his/her own abscissas and weights for a given integral [30], it is often convenient to use the well-known orthogonal polynomials [31], such as the Legendre, Chebyshev, or Laguerre to obtain these [30]. In this paper, we have used the Gauss-Legendre quadrature [8] formula for which the weight function $W(x)$ is 1 , and the domain of the independent variable $x$ is $(-1,1)$. A mapping is used to set the domain of $\theta$ and $\phi$ to $(-1,1)$.

The discrete ordinates method is a technique used to replace an integro-differential equation by a system of ordinary differential equations [27]. Once the integral is approximated by Gaussian quadrature, the integro-differential equation may be written as a set of uncoupled ordinary differential equations, where each equation corresponds to each abscissa [8], [26], [27].

Application of the discrete ordinates method to (5) will result in a set of uncoupled equations, one for each quadrature point, i.e.,

$$
\begin{aligned}
& u \frac{\partial}{\partial \xi} I\left(\xi, u, \phi_{r}, \tau\right)+\sigma_{t} I\left(\xi, u, \phi_{r}, \tau\right) \\
& -\frac{\sigma_{s}}{4 \pi} \sum_{j=1}^{L} w_{j}^{\phi} \int_{-1}^{1} P\left(u^{\prime}, \phi_{j} ; u, \phi_{r}\right) I\left(\xi, u^{\prime}, \phi_{j}, \tau\right) d u^{\prime}=0,
\end{aligned}
$$

where $r=1, \ldots, L$. The original four-variable RTE has therefore been reduced to a three-variable integro-differential equation but with only a simple integral instead of the double integral in the original equation. In (7), $\phi_{r}$ is the $r$ th quadrature point and $w_{j}^{\phi}$ is the corresponding Gaussian weight.
The next section discusses the removal of the time dependency of the aforementioned formulation.

\section{B. Removal of the Time Dependence}

The time dependency is represented using the Laguerre expansion. Laguerre polynomials are the canonical solutions of the differential equation

$$
x y^{\prime \prime}+(1-x) y^{\prime}+n y=0
$$

which is also known as the Laguerre's equation [28]. These solutions are a sequence of orthogonal polynomials that can be generated by Rodrigues' formula [28]

$$
L_{n}(x)=\frac{e^{x}}{n !} \frac{d^{n}}{d x^{n}}\left(e^{-x} x^{n}\right) .
$$

These polynomials satisfy the orthogonality property

$$
\int_{0}^{\infty} L_{n}(x) L_{m}(x) e^{-x} d x=\delta_{n m}
$$

where $\delta_{n m}$ is the Kronecker delta [32]. Laguerre polynomials are causal [33], and thus, the causality of the system is implicitly imposed. Also, any causal function can be expanded using a Laguerre basis because it forms a complete orthogonal basis in real space [31]. Since such expansions are a linear superposition of causal Laguerre polynomials, the causality property of the original system is implicitly retained.

We expand the intensity as a summation of $N$ Laguerre polynomials;

$$
I(\xi, u, \tau)=\sum_{k=0}^{N} B_{k}(\xi, u) L_{k}(\tau)
$$

where

$$
\int_{0}^{\infty} L_{n}(\tau) L_{m}(\tau) e^{-\tau} d \tau=\delta_{m n} .
$$

Using (11) in (7) and taking moments [i.e., multiplying by $L_{n}(\tau) e^{-\tau}$ and integrating over $\left.[0, \infty)\right]$, the time dependence can be removed as shown next

$$
\begin{aligned}
& u \frac{\partial}{\partial \xi} B_{n}\left(\xi, u, \phi_{r}\right)+\sigma_{t} B_{n}\left(\xi, u, \phi_{r}\right) \\
& -\frac{\sigma_{s}}{4 \pi} \sum_{j=1}^{L} w_{j}^{\phi} \int_{-1}^{1} P\left(u^{\prime}, \phi_{j} ; u, \phi_{r}\right) B_{n}\left(\xi, u^{\prime}, \phi_{j}\right) d u^{\prime}=0 .
\end{aligned}
$$

Similar operations should be carried out on the boundary condition. Thus, (2) can be written in the form

$$
\sum_{k=0}^{N} B_{k}(\xi=0, u, \phi) L_{k}(\tau)=\sum_{k=0}^{N} C_{k} \delta\left(u-u_{0}\right) \delta\left(\phi-\phi_{0}\right) L_{k}(\tau)
$$

where

$$
C_{k} \approx \sum_{j=1}^{q} w_{j} f\left(\tau_{j}\right) L_{k}\left(\tau_{j}\right)
$$

The boundary condition (14) should be satisfied for all $\tau$. Hence, in (14), each coefficient on the left-hand side is equal to that of 
the right-hand side, i.e.

$$
B_{k}(\xi=0, u)=C_{k} \delta\left(u-u_{0}\right) \delta\left(\phi-\phi_{0}\right)
$$

Still, the reduced RTE, (13), is an integro-differential equation but with only two independent variables $\xi$ and $u$. The next step is to replace the integral term by a summation using discrete ordinates method for $u$ as detailed later.

\section{Discretization of the Zenith Angle}

The discrete ordinates method [8], [26], [27] is used for this purpose as for the azimuthal discretization. Thus, the integral term in (13) is approximated by a summation using Gaussian quadrature [8], [30]. It is then followed by replacing (13) by the set of equations

$u_{i} \frac{\partial}{\partial \xi} B_{n}\left(\xi, u_{i}, \phi_{r}\right)+\sigma_{t} B_{n}\left(\xi, u_{i}, \phi_{r}\right)$

$-\frac{\sigma_{s}}{4 \pi} \sum_{j=1}^{L} w_{j}^{\phi} \sum_{k=1}^{K} w_{k}^{u} P\left(u_{k}, \phi_{j} ; u_{i}, \phi_{r}\right) B_{n}\left(\xi, u_{k}, \phi_{j}\right)=0$

where $i=0, \ldots, K, r=0, \ldots, L$, and $n=0, \ldots, N$.

In (17), $u_{i}$ is the $i$ th quadrature point and $w_{i}^{u}$ is the corresponding Gaussian weight. There are $K$ number of uncoupled equations corresponding to each quadrature point of the azimuthal angle $\phi_{r}$. Considering all the quadrature points for the azimuthal angle, there is a set of $L$ equations similar to (17), which represents the $r$ th equation of this set. Therefore, it is possible to combine this set and write it in matrix form as next

$$
\frac{\partial}{\partial \xi} \boldsymbol{\Lambda} \mathbf{B}_{n}+\sigma_{t} \mathbf{B}_{n}-\frac{\sigma_{s}}{4 \pi} \mathbf{P} \mathbf{W B} \mathbf{B}_{n}=\mathbf{0}
$$

where $n=0, \ldots, N, \mathbf{B}_{n}=\left[\mathbf{B}_{n}\left(\xi, u_{i}, \phi_{r}\right)\right]_{(K \times L), 1}, \mathbf{P}=\left[P\left(u_{k}\right.\right.$, $\left.\left.\phi_{j} ; u_{i}, \phi_{r}\right)\right]_{(K \times L),(K \times L)}$, and $\boldsymbol{\Lambda}$ is a $(K \times L)$ by $(K \times L)$ diagonal matrix with diagonal elements $u_{1}$ to $u_{K}$ repeating $L$ times. The matrix $\mathbf{W}$ is also a $(K \times L)$ by $(K \times L)$ diagonal matrix with diagonal elements $w_{r}^{\phi} \times w_{k}^{u}$ with the pattern $w_{1}^{\phi} \times w_{1}^{u}, w_{1}^{\phi} \times w_{2}^{u}, \ldots, w_{1}^{\phi} \times w_{K}^{u}, w_{2}^{\phi} \times w_{1}^{u}, \ldots, w_{L}^{\phi} \times w_{K}^{u}$.

Rearranging, (18) can be written as

$$
\frac{\partial}{\partial \xi} \mathbf{B}_{n}=\mathbf{\Gamma} \mathbf{B}_{n}
$$

where

$$
\boldsymbol{\Gamma}=\boldsymbol{\Lambda}^{-1}\left[\frac{\sigma_{s}}{4 \pi} \mathbf{P} \mathbf{W}-\sigma_{t} \mathbf{I}\right] .
$$

Hence, the original RTE is reduced to a one-variable ordinary differential equation. The boundary condition given by (16) is simplified in a similar fashion, which results in

$\mathbf{B}_{n}(\xi=0)= \begin{cases}C_{k}, & \text { for } u=u_{0} \text { and } \phi=\phi_{0} \\ 0, & \text { for } u \neq u_{0} \text { or } \phi \neq \phi_{0}\end{cases}$

Thus, (19), subject to the aforementioned boundary condition, can be solved using the fourth-order RKF method [29]. We have chosen the fourth-order RKF because a compromise should be made between the accuracy and the execution time of the algorithm. However, for applications where very high precision is essential, a higher order RKF method may be used. The RKF method can be used to find the solution to an ordinary differential equation numerically, at a given value of the independent variable, provided that the initial condition is given. This method uses adaptive step sizes, and the error at each step is estimated as the difference between the fifth- and fourth-order estimates [29].

The next section presents some results obtained using this algorithm and a discussion on relative advantages of the proposed algorithm.

\section{NUMERICAL RESULTS AND DISCUSSION}

We used Matlab for implementing the proposed solution strategy for the RTE. It is interesting to make the following note about the phase function used in our numerical simulations. Even though our algorithm does not depend on the specific nature of the phase function, it has significant influence on the interpretation of results. The exact phase function in biological tissue is still a research issue [34]. In the past, the Henyey-Greenstein phase function (HGPF) was widely used for modeling radiative transfer in many applications [34], [35]. However, due to some discrepancies, recently, researchers have combined a highly anisotropic phase function [(e.g., the Henyey-Greenstein phase function)], and a low anisotropic phase function [e.g., the Rayleigh phase funcition (RLPF)] [36] to approximate the phase function in biological tissue [36]. The HGPF represents large particles and the RLPF represents small particles compared to the wavelength of the incident light [36]. Bevilacqua et al. [36] used the following phase function to model biological tissue

$$
P_{\text {tissue }}(\cos \Theta)=(1-\alpha) P_{\mathrm{HG}}(\cos \Theta)+\alpha P_{\mathrm{RL}}(\cos \Theta) \text {, }
$$

where

$$
P_{\mathrm{HG}}(\cos \Theta)=\frac{1-g^{2}}{2\left(1+g^{2}-2 g \cos \Theta\right)^{3 / 2}}
$$

is the Henyey-Greenstein phase function, where $g$ is the asymmetry factor and $\Theta$ is the angle between incoming and scattered rays

$$
\begin{gathered}
\left(\cos \Theta=\cos \theta^{\prime} \cos \theta+\sin \theta^{\prime} \sin \theta \cos \left(\phi^{\prime}-\phi\right)\right. \\
\left.=u^{\prime} u+\sqrt{\left(1-u^{\prime 2}\right)} \sqrt{\left(1-u^{2}\right)} \cos \left(\phi^{\prime}-\theta\right)\right) \\
P_{\mathrm{RL}}(\cos \Theta)=\frac{3}{8}\left(1+\cos ^{2} \Theta\right)
\end{gathered}
$$

is the Rayleigh phase function [35], [36]. In [22], the value of $\alpha$ depends on the properties of the tissue specimen [36]. The solution technique we propose is independent of the selection of the phase function. To illustrate this idea, we have included simulation results for all the aforementioned three phase functions.

Also, the input pulse was taken to be a Gaussian pulse given by

$$
f(t)=I_{0} e^{-\left(\frac{\left(t-t_{0}\right)}{T}\right)^{2}}
$$

\section{A. Normalization of the Units}

The RTE, (1), is linear in intensity $I$, and thus representing $I$ as $I / I_{0}$ does not change the equation. Therefore, we use an arbitrary scale for $I$ throughout this paper. The time units are 


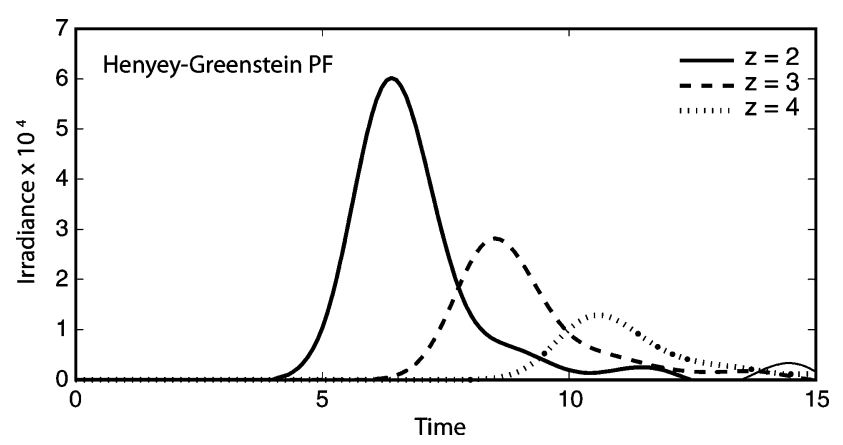

Fig. 3. Variation of the irradiance with time at different locations along the $\mathrm{z}$-axis.

normalized by $T_{s}$, spatial units by $Z_{s}$, and scattering and absorption coefficients by $1 / Z_{s}$, unless specified otherwise. With this normalization, (1) can be written as

$$
\begin{array}{r}
\frac{\partial}{\partial \widetilde{t}} \widetilde{I}(\widetilde{z}, u, \phi, \widetilde{t})+u \frac{\partial}{\partial \widetilde{z}} \widetilde{I}(\widetilde{z}, u, \phi, \widetilde{t}) \\
-\frac{\widetilde{\sigma_{s}}}{4 \pi} \int_{0}^{2 \pi} \int_{-1}^{1} P\left(u^{\prime}, \phi^{\prime} ; u, \phi\right) \widetilde{I}\left(\widetilde{z}, u^{\prime}, \phi^{\prime}, \widetilde{t}\right) d u^{\prime} d \phi^{\prime} \\
+\widetilde{\sigma_{t}} \widetilde{I}(\widetilde{z}, u, \phi, \widetilde{t})=0 .
\end{array}
$$

In this paper, we have set $T_{s}=T$, where $T$ is the factor determining the width of the input pulse. We choose this normalization factor due to the fact that the Laguerre approximation of the Gaussian pulse is very accurate for pulses with $T=1$ or greater. Therefore, with this scaling, it is possible to obtain very accurate results even for very narrow pulses, which are used in many biomedical applications. For pulses with other shapes, it is recommended that a least square fit is used to obtain a Gaussian approximation, and then, set $T_{s}$ to be the width of that Gaussian pulse.

We have set $Z_{s}=v \times \bar{T}$, here, $\bar{T}$ can be chosen to suit the particular application. However, these scaling factors should be chosen carefully so that the matrices remain well conditioned. For the simulations presented in this paper, without loss of generality, we have chosen $T / \bar{T}=1$ so that $Z_{s}=v \times T$.

Also, the classical Laguerre polynomials given in (9) tends to infinity at a very high rate for $x>10$. Thus, the approximation of a pulse at $\widetilde{z}=0$ will be accurate only in the domain $[0,10]$. However, since in this algorithm, the Laguerre polynomials are propagated with the pulse, the approximation will always be accurate in the domain $[0, a]$, where $a=10+\widetilde{z} /(v u)$, i.e., the domain in which the Laguerre fit is accurate extends by up to 10 time units beyond the required observation point.

Fig. 3 shows the variation of the irradiance with time taken at different locations along the $z$-axis. For this figure, the absorption coefficient $\widetilde{\sigma_{a}}$ was taken to be 0.002 and the scattering coefficient $\widetilde{\sigma_{s}}$ was kept at 0.988 . As $\widetilde{z}$ increases, the peak value of the irradiance drops and the pulse is shifted in time by the amount $\left(t-t_{0}-\frac{z}{v u}\right)$. The Henyey-Greenstein phase function with $g=0.7$ was used for this simulation.

Fig. 4 shows the variation of the irradiance with time at $\widetilde{z}=2$ for different values of $\widetilde{\sigma_{a}}$. It can be seen that as $\widetilde{\sigma_{a}}$ increases, the peak value of the irradiance drops. For this set of plots, $\widetilde{\sigma_{s}}$ was

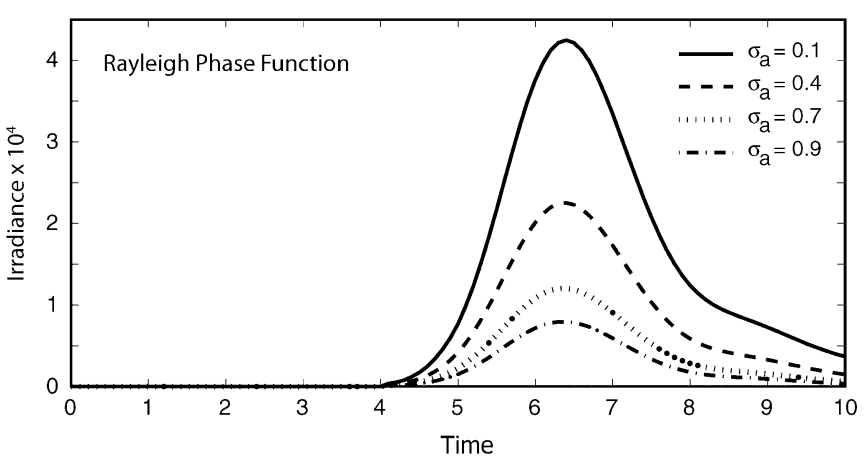

Fig. 4. Variation of the irradiance with time at $z=2$ with different values of the absorption coefficient.

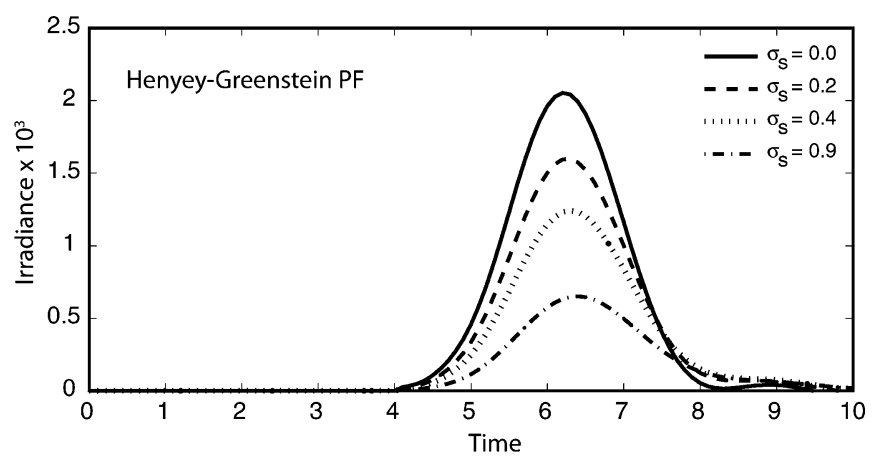

Fig. 5. Variation of the irradiance with time at $z=2$ with different values of the scattering coefficient.

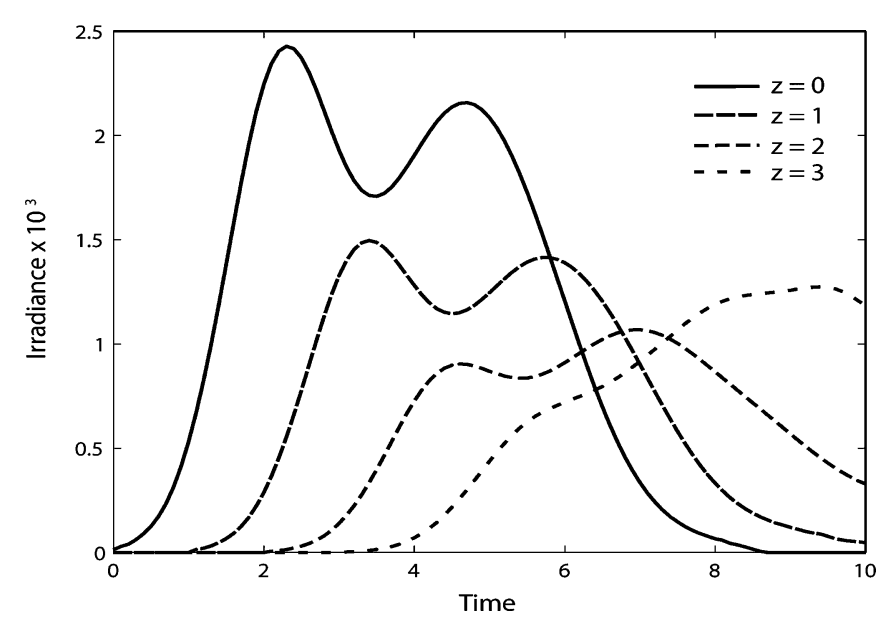

Fig. 6. Variation of the irradiance with $z$ for an arbitrary input.

kept constant at 0.9 and the Rayleigh phase function was used. Fig. 5 shows the variation of the irradiance with time at $\widetilde{z}=2$ for different values of $\widetilde{\sigma_{s}}$. It is evident that as $\widetilde{\sigma_{s}}$ increases, the peak value of the irradiance drops. For this case, $\widetilde{\sigma_{a}}$ was kept constant at 0.02 , and again, the Henyey-Greenstein phase function is used with $g=0.7$.

Fig. 6 shows the variation of the intensity at different $\widetilde{z}$ values for an arbitrary shaped pulse with the same phase function. 


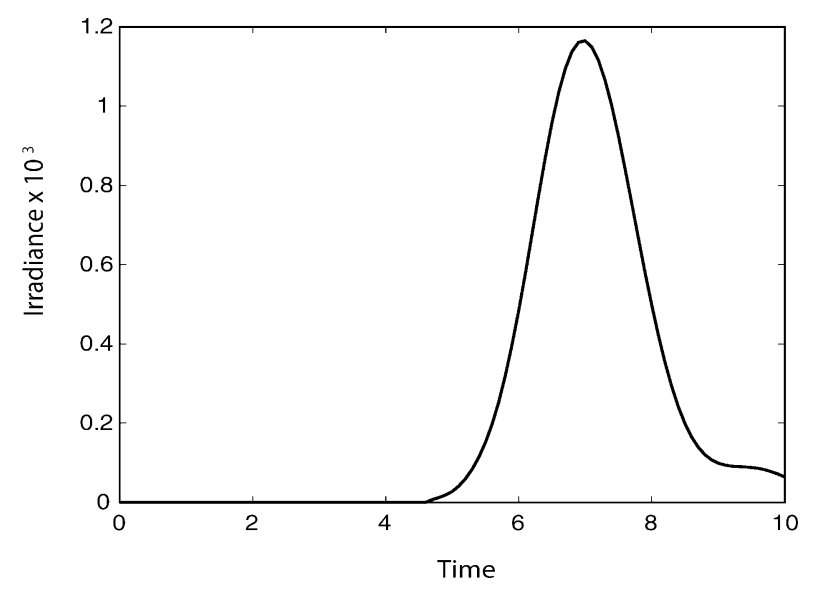

Fig. 7. Variation of the irradiance with time and $u$ for a human skin specimen.

In the aforementioned figures, the values for scattering and absorption coefficients were arbitrarily selected in order to demonstrate the proposed algorithm. Fig. 7 shows the variation of intensity for a human skin specimen with an incident pulse of the form (25) with $T=1 \mathrm{ps}, \sigma_{a}=0.5 \mathrm{~mm}^{-1}$, and $\sigma_{s}=0.8 \mathrm{~mm}^{-1}$ [37]. Also, for this figure, $v=0.215 \mathrm{~mm} / \mathrm{ps}$, and the plot was obtained at $z=1 \mathrm{~mm}$. We have not normalized the time, spatial units, or scattering and absorption coefficients for this figure, and have used data for a human skin specimen. However, the irradiance has the same unit as that of the input pulse $I_{0}$. For this simulation, the combined phase function $P_{\text {tissue }}(\cos \Theta)$ [36] was used.

The aforementioned results were obtained using 63 Laguerre modes, 15 quadrature points for the zenith angle $\theta$, and 20 quadrature points for the azimuthal angle $\phi$. The extension of the proposed technique for inhomogeneous media is straightforward as detailed in the previous section. Also, it can be easily seen that this method can be used to solve the 2-D and 3-D RTE with only slight modifications to the formulation.

\section{B. Extension To Inhomogeneous Media}

The proposed formulation can be easily extended for inhomogeneous media. In an inhomogeneous medium, the scattering and absorption coefficients are a function of $z$. The RTE for this case is then

$$
\begin{aligned}
& \frac{1}{v} \frac{\partial}{\partial t} I(z, u, \phi, t)+ u \frac{\partial}{\partial z} I(z, u, \phi, t) \\
&-\frac{\sigma_{s}(z)}{4 \pi} \int_{0}^{2 \pi} \int_{-1}^{1} P\left(u^{\prime}, \phi^{\prime} ; u, \phi\right) I\left(z, u^{\prime}, \phi^{\prime}, t\right) d u^{\prime} d \phi^{\prime} \\
& \quad+\sigma_{t}(z) I(z, u, \phi, t)=F(z, u, \phi, t) . \quad(27)
\end{aligned}
$$

If we go through all the steps in the proposed algorithm, (27) will be reduced to

$$
\frac{\partial}{\partial \xi} \mathbf{B}_{n}(\xi)=\boldsymbol{\Gamma}(\xi) \mathbf{B}_{n}(\xi)
$$

where

$$
\boldsymbol{\Gamma}=\boldsymbol{\Lambda}^{-1}\left[\frac{\sigma_{s}(\xi)}{4 \pi} \mathbf{P} \mathbf{W}-\sigma_{t}(\xi) \mathbf{I}\right]
$$

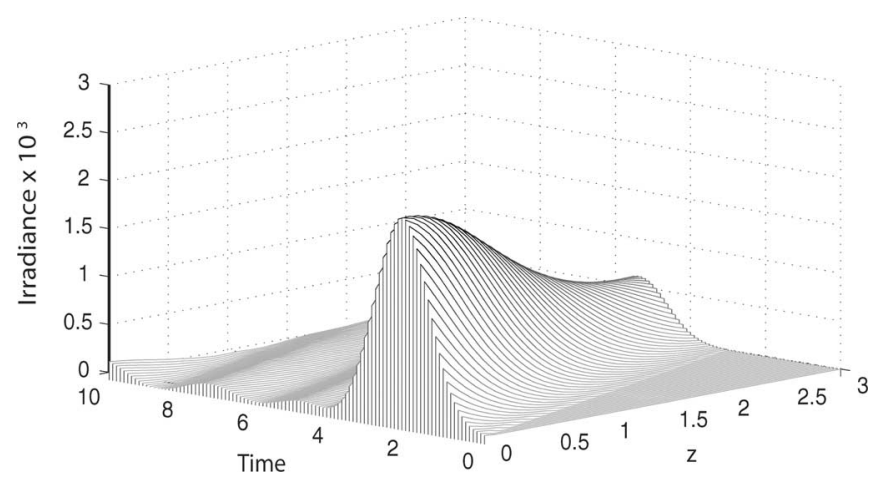

Fig. 8. Variation of the irradiance with time and distance in an inhomogeneous medium.

Thus, (28), subject to the boundary conditions, can be solved using the RKF method as described previously. Fig. 8 shows the irradiance variation with time and distance in an inhomogeneous medium with $\widetilde{\sigma_{a}}=0.05, \widetilde{\sigma_{s}}=\sin (z) \times e^{-z / 5}$, and Rayleigh scattering. As explained earlier, an extension to inhomogeneous media is straight forward.

\section{Refractive Index-Mismatched Interfaces}

If the refractive index mismatch between the tissue and air interface is considered, the specular and diffuse reflectivities of the interface should be incorporated into the boundary conditions of the radiative transfer equation. The specular reflectivity at the interface can be calculated from Fresnel's equations, and the diffuse reflectivity may assumed to be uniform [38]. Our proposed method can incorporate such changes in boundary conditions easily because our numerical integration strategy is independent of the format of the boundary conditions because the zenith and azimuthal angle dependencies can be removed by the discrete ordinate method, and Laguerre series can represent the time evolution.

\section{Computational Complexity}

The proposed algorithm involves only one matrix inversion, which is a diagonal matrix. The size of this matrix and other matrices depend on the product of the number of quadrature points for $\phi$ and $u$. These numbers should be chosen according to the required resolution of the application. Also, there is one matrix multiplication and one matrix addition. The RKF method involves only six function evaluations in each step. If $\Gamma$ in (19) is made a scalar (i.e., if the number of quadrature points for $\phi$ and $u$ is set to 1 ), the RKF routine takes around 100 steps to solve for a unit distance along the $z$-axis (i.e., for $\widetilde{z}=1$ ). Doubling the distance requires only 20 additional steps. If $\boldsymbol{\Gamma}$ in (19) is set to a $16 \times 16$ matrix (by taking four quadrature points for each angle), the number of steps required would be around 200 . Doubling the distance in this case requires only 60 additional steps. 


\section{CONCLUSION}

This paper introduced a novel efficient approach for solving the radiative transfer equation, which governs the light propagation through biological tissue. The proposed method used a Laguerre approximation to represent the time dependence and discrete ordinates method to discretize the azimuthal and zenith angles. This reduced the original integro-differential equation to a single-variable ordinary differential equation, which was then solved using the fourth-order RKF method.

There are a number of advantages of this technique over most of the other existing methods. Since the time dependence is expanded using a Laguerre basis, all the sampling points in the time domain is obtained in a single execution, as opposed to time-marching techniques used in existing solution methods. This makes the proposed algorithm much faster when one requires the intensity profile at a particular point or a plane over a time interval. The incident pulse can be approximated using a relatively small number of Laguerre polynomials. The causality of the system is implicitly imposed by the causal Laguerre polynomials. Any causal function can be expanded using Laguerre polynomials because it forms a complete orthogonal basis in real space. Since such expansions are a linear superposition of causal Laguerre polynomials, the causality property of the original system is implicitly retained. The use of the RKF method for the spatial variable $z$ makes the extension to inhomogeneous media simple and straightforward as detailed earlier.

\section{REFERENCES}

[1] F. Liu, K. M. Yoo, and R. R. Alfano, "Ultrafast laser-pulse transmission and imaging through biological tissues," Appl. Opt., vol. 32, pp. 554-558, 1993.

[2] G. Yao and L. V. Wang, "Theoretical and experimental studies of ultrasound-modulated optical tomography in biological tissue," Appl. Opt., vol. 39, pp. 659-664, 2000.

[3] S. B. Colak, M. B. van der Mark, G. W. 't Hooft, J. H. Hoogenraad, E. S. van der Linden, and F. A. Kuijpers, "Clinical optical tomography and NIR spectroscopy for breast cancer detection," IEEE J. Sel. Topics Quantum Electron., vol. 5, no. 4, pp. 1143-1158, Jul./Aug. 1999.

[4] B. D. Cameron, H. W. Gorde, B. Satheesan, and G. L. Cote, "The use of polarized laser light through the eye for noninvasive glucose monitoring," Diabetes Tech. Ther., vol. 1, pp. 135-143, 1999.

[5] A. D. Klose, U. Netz, J. Beuthan, and A. H. Hielscher, "Optical tomography using the time-independent equation of radiative transfer-Part 1 : Forward model," J. Quantum Spectrosc. Radiat. Trans., vol. 72, pp. 691$713,2002$.

[6] K. Mitra and S. Kumar, "Development and comparison of models for light-pulse transport through scattering-absorbing media," Appl. Opt., vol. 38, pp. 188-196, 1999.

[7] M. Premaratne, E. Premaratne, and A. J. Lowery, "The photon transport equation for turbid biological media with spatially varying isotropic refractive index," Opt. Exp., vol. 13, pp. 389-399, 2005.

[8] K. Stamnes, S. C. Tsay, W. Wiscombe, and K. Jayaweera, "Numerically stable algorithm for discrete-ordinate-method radiative transfer in multiple scattering and emitting layered media," Appl. Opt., vol. 27, pp. 2502 2509,1988

[9] J. L. Deuzé, M. Herman, and R. Santer, "Fourier series expansion of the transfer equation in the atmosphere-ocean system," J. Quantum Spectrosc. Radiat. Trans., vol. 41, pp. 483-494, 1989.

[10] Z. Jin and K. Stamnes, "Radiative transfer in nonuniformly refracting layered media: atmosphere-ocean system," Appl. Opt., vol. 33, pp. 431442, 1994.

[11] C. E. Siewert and J. R. Thomas, "Radiative transfer calculations in spheres and cylinders," J. Quantum Spectrosc. Radiat. Transfer, vol. 34, pp. 5964, 1985 .
[12] C. E. Siewert, "A radiative-transfer inverse-source problem for a sphere," J. Quantum Spectrosc. Radiat. Transfer, vol. 52, pp. 157-160, 1994.

[13] G. C. Pomraning and G. M. Foglesong, "Transport-diffusion interfaces in radiative transfer," J. Comput. Phys., vol. 32, pp. 420-436, 1979.

[14] E. W. Larsen, "The inverse source problem in radiative transfer," $J$. Quantum Spectrosc. Radiat. Transfer, vol. 15, pp. 1-5, 1975.

[15] O. Dorn, "A transport-backtransport method for optical tomography," Inverse Probl., vol. 14, pp. 1107-1130, 1998.

[16] E. W. Larsen, G. C. Pomraning, and V. C. Badham, "Asymptotic analysis of radiative transfer problems," J. Quantum Spectrosc. Radiat. Trans., vol. 29, pp. 285-310, 1983.

[17] T. Tarvainen, M. Vauhkonen, V. Kolehmainen, and J. P. Kaipio, "Finite element model for the coupled radiative transfer equation and diffusion approximation," Int. J. Numer. Methods Eng., vol. 65, pp. 383-405, 2006.

[18] M. Sakami, K. Mitra, and P. Hsu, "Analysis of light pulse transport through two-dimensional scattering and absorbing media.," J. Quantum Spectrosc. Radiat. Trans., vol. 73, pp. 169-179, 2002.

[19] Z. M. Tan and P. F. Hsu, "An integral formulation of transient radiative transfer," Trans. ASME, vol. 123, pp. 466-475, 2001.

[20] A. D. Kim and A. Ishimaru, "A Chebyshev spectral method for radiative transfer equations applied to electromagnetic wave propagation and scattering in discrete random media," J. Comput. Phys., vol. 152, pp. 264-280, 1999.

[21] A. D. Kim and M. Moscoso, "Chebyshev spectral methods for radiative transfer," SIAM J. Sci. Comput., vol. 23, pp. 2074-2094, 2002.

[22] J. V. P. de Oliveira, A. V. Cardona, and M. T. M. B. de Vilhena, "Solution of the one-dimensional time-dependent discrete ordinates problem in a slab by the spectral and LTSN methods," Ann. Nucl. Energy, vol. 29, pp. 13-20, 2002

[23] J. V. P. de Oliveira, A. V. Cardona, M. T. Vilhena, and R. C. Barros, "A semi-analytical numerical method for time-dependent radiative transfer problems in slab geometry with coherent isotropic scattering," J. Quantum Spectrosc. Radiat. Trans., vol. 73, pp. 55-62, 2002.

[24] J. A. Fleck, "The calculation of nonlinear radiation transport by a Monte Carlo method," in Methods in Computational Physics, vol. 1, New York: Academic, 1963, pp. 43-65.

[25] A. B. Carlson, "Signals and Spectra," in Communication Systems: An Introduction to Signals and Noise in Electrical Communication, 3rd ed. Singapore: McGraw-Hill, 1986, pp. 53-55.

[26] S. Chandrasekhar, "Quadrature formulae," in Radiative Transfer, New York: Dover, 1960, pp. 54-60.

[27] K. Stamnes and R. A. Swanson, "A new look at the discrete ordinate method for radiative transfer calculations in anisotropically scattering atmospheres," J. Atmos. Sci., vol. 38, pp. 387-399, 1981.

[28] M. Abramomitz and I. A. Stegun, "Orthogonal polynomials," in Handbook of Mathematical Functions with Formulas, Graphs and Mathematical Tables, New York: Dover, 1965, pp. 773-784.

[29] S. C. Chapra and R. P. Canale, "Runge-Kutta methods," in Numerical Methods for Engineers, 4th ed. New York: McGraw-Hill, 2002, pp. 719 720.

[30] W. H. Press, S. A. Teukolsky, W. T. Vetterling, and B. P. Flannery, "Integration of functions," in Numerical Recipes in $\mathrm{C}++, 2$ nd ed. New York: Cambridge Univ. Press, 2003, pp. 152-157.

[31] G. A. Korn and T. M. Korn, "Special functions," in Mathematical Handbook for Scientists and Engineers: Definitions, Theorems and Formulas for Reference and Review, 2nd ed. New York: Dover, 2000, pp. 848-856.

[32] G. A. Korn and T. M. Korn, "Tensor analysis," in Mathematical Handbook for Scientists and Engineers: Definitions, Theorems and Formulas for Reference and Review, 2nd ed. New York: Dover, 2000, p. 544.

[33] C. J. Rivero-Moreno and S. Bres, "Video spatio-temporal signatures using polynomial transforms," Lect. Notes Comput. Sci., vol. 3736, pp. 50-59, 2005.

[34] S. K. Sharma and S. Banerjee, "Role of approximate phase functions in Monte Carlo simulation of light propagation in tissues," J. Opt. A: Pure Appl. Opt., vol. 5, pp. 294-302, 2003.

[35] G. E. Thomas and K. Stamnes, Radiative Transfer in the Atmosphere and Ocean. Cambridge: Cambridge Univ. Press, 1999.

[36] F. Bevilacqua, D. Piguet, P. Marquet, J. D. Gross, B. J. Tromberg, and C. Depeursinge, "In vivo local determination of tissue optical properties: Applications to human brain," Appl. Opt., vol. 38, pp. 4939-4950, 1999.

[37] T. L. Troy and S. N. Thennadil, "Optical properties of human skin in the near infrared wavelength range of 1000 to $2200 \mathrm{~nm}$, , J. Biomed. Opt., vol. 6, pp. 167-176, 2001.

[38] A. R. Degheidy and M. S. A. Krim, "Effects of Fresnel and diffused reflectivities on light transport in a half-space medium," J. Quantum Spectrosc. Radiat. Transf., vol. 61, pp. 751-757, 1999. 


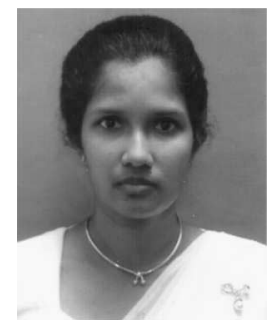

Chintha C. Handapangoda ( $\left.\mathrm{S}^{\prime} 07\right)$ received the B.Sc. (Eng.) degree in electrical and electronic engineering with a first-class honors from the University of Peradeniya, Peradeniya, Sri Lanka, in 2005. She is currently working toward the Ph.D. degree in biomedical engineering at Monash University, Clayton, Vic., Australia.

She was a Pre-University Research Assistant in the Photochemistry Division at the Instititue of Fundamental Studies, Sri Lanka, in 2000, an undergraduate trainee at the Arthur C Clark Institute for Modern Technologies, Colombo, Sri Lanka, in 2003, and an Instructor for the Department of Engineering Mathematics at the University of Peradeniya, in 2005.

Ms. Handapangoda is an Associate Member of the Institution of Engineers, Colombo, Sri Lanka (IESL). She was the recipient of gold medal and a prize for the best performance in electrical and electronic engineering together with a prize for electronic communications, in 2005.

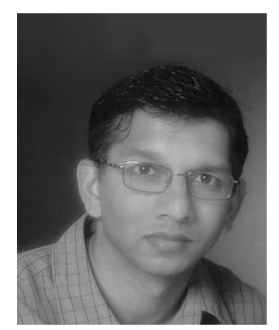

Malin Premaratne (M'98-SM'03) received the B.Sc. (Maths) and B.E. (Elec.) degrees, both with first-class honors, and the Ph.D. degree from the University of Melbourne, Melbourne, Vic., Australia, in 1995 and 1998, respectively.

From 1998 to 2000, he was with the Photonics Research Laboratory, a division of the Australian Photonics Cooperative Research Centre (APCRC), University of Melbourne, where he was the CoProject Leader of the APCRC Optical Amplifier Project. During this period, he was associated with Telstra, Australia and Hewlett Packard, through the University of Melbourne. From 2000 to 2003, he was associated with several leading startups in the photonic area either as an employee or a Consultant. During this period, he was on the editorial boards of the SPIE/Kluwer and Wiley Publishers in the optical communications area. From 2001 to 2003, he worked as a Product Manger (Research and Development) of VPI systems, Optical Systems Group, Holmdel, NJ. Since 2003, he has brought forward the research program in high-performance computing applications to complex systems simulations at the Advanced Computing and Simulation Laboratory (AXL), Monash University, Clayton, Vic., where he is currently a Deputy Research Director. He is the author or coauthor of more than 100 published research papers in the areas of semiconductor lasers, erbium-doped fiber amplifier (EDFA) and Raman amplifiers, optical network design algorithms, and numerical simulation techniques. He has held visiting research appointments with the University of Melbourne, the Australian National University, the University of California-Los Angeles (UCLA), and the University of Rochester, New York.

Dr. Premaratne is a Fellow of the Institution of Engineers, Australia (FIEAust). He is also an Executive Member of the IEAust, Vic. Since 2001, he has been the Chairman of the IEEE Lasers and the Electro-Optics Society.

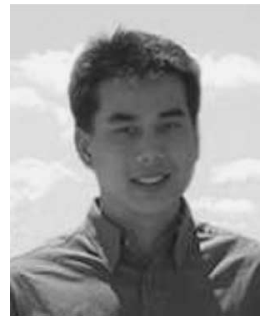

Leslie Yeo received the M.Eng. and Ph.D. degrees in chemical engineering from the Imperial College, London, U.K., in 1998 and 2002, respectively.

He was a Postdoctoral Researcher at the University of Notre Dame, Notre Dame, IN, in 2003-2004, thereafter, he was in the industry as a Mathematical Modeller at Det Norske Veritas, Oslo, Norway, in 2002-2003. Currently, he is a Senior Lecturer in the Department of Mechanical Engineering, Monash University, Clayton, Vic., Australia. His current research interests include primarily the microscale and nanoscale phenomena, and, in particular, microfluidics.

Dr. Yeo is a Member of the Editorial Board of Biomicrofluidics published by the American Institute ofPhysics. He was the recipient of the Dudley Newitt Prize for a theoretical/computational thesis of outstanding merit.

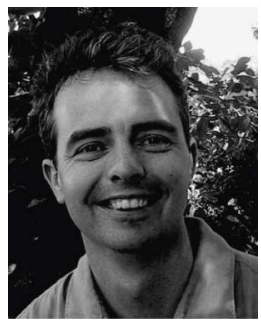

James Friend (S'98-A'98-M'02) received the B.S. degree in aerospace engineering and the M.S. and $\mathrm{Ph} . \mathrm{D}$. degrees in mechanical engineering from the University of Missouri-Rolla, Rolla, MO, in 1992, 1994, and 1998, respectively.

From 2001 to 2004, he was an Assistant Professor at the Precision and Intelligence Laboratory, Tokyo Institute of Technology, Tokyo, Japan. Currently, he is an Associate Professor in the Department of Mechanical Engineering, Monash University, Clayton, Vic., Australia. His current research interests include bio-, micro-nanodevices. He was the recipient of several awards including the ASME Structures and Materials Award and an AIAA Jefferson Goblet Student Award for a paper on ultrasonic motor analysis in 1996, an award for the encouragement of young scientists at the Symposium for Ultrasonic Electronics and Engineering in 2003, for a presentation on acoustic waveguides, and an award in 2004, for a presentation on the Scream Actuator atthe Spring Meeting of the Acoustical Society of Japan.

Dr. Friend is a Member of the American Society of Mechanical Engineering (ASME), and a Committee Member of the IEEE Nanotechnology for Biology Society. 